\title{
Study of the Intrinsic Viscosity[ $\eta]$ of Poly(methyl methacrylate) Solution by QSPR
}

\author{
WISAM A RADHI ${ }^{1}$, SADIQ M-H ISMAEL ${ }^{2}$ and KAWKAB A HUSSAIN ${ }^{2}$ \\ ${ }^{1}$ Department of Chemistry, Polymer Research Center, University of Basrah, Iraq \\ ${ }^{2}$ Department of Chemistry, College of Education for Pure Science, \\ University of Basrah, Iraq \\ kawkabali@ymail.com
}

Received 21 October 2016 / Accepted 6 Noveber 2016

\begin{abstract}
Quantitative structure-property relationship (QSPR) analysis to intrinsic viscosity [ $\eta]$ of poly(methyl methacrylate) solution have been conducted. The study was done by using molecular modeling. The calculation was performed by the PM7 method using MOPAC programme. The relationship analysis between intrinsic viscosity $[\eta]$ and physicochemical properties of eight solvents (toluene, ethyl acetate, benzene, chloroform, butanone, dichloroethane, acetone and nitro ethane) under study was done by MLR analysis to generate the equation that relates the structural features to the intrinsic viscosity [ $\eta$ ] properties. The results show excellent models with four parameters linear equations. The best model using theoretical parameters is identified, that including electronic energy, $\omega$, dielectric energy and energy gap parameters, with excellent statistical fit as evident from its $\mathrm{R}^{2}=0.993, \mathrm{~F}=107.222$ and $\mathrm{S}=3.447$.
\end{abstract}

Keywords: QSPR, Poly(methyl methacrylate), Intrinsic viscosity

\section{Introduction}

The design of new materials with optimal thermophysical, mechanical and optical properties is a challenge for computational chemistry. Novel materials are typically developed using a trial and error approach, which is costly and time consuming ${ }^{1}$. An alternative strategy is to model the material properties as functions of the molecular structure using the so called quantitative structure-property relationships $(\mathrm{QSPR})^{2,3}$. Application of QSPR methodologies in material design has the potential to decrease considerably the time and effort required to improve material properties in terms of their efficacy or to discover new materials with desired properties ${ }^{4}$. The QSPR approach is based on the assumption that the variation of the behavior of the compounds, as expressed by any measured physicochemical properties, can be correlated with changes in molecular features of the compounds termed descriptors ${ }^{5,6}$. The advantage of this approach lies in the fact that it requires only the knowledge of the chemical structure and is not dependent on any experimental properties. The QSPR approach has been successfully used to predict many polymeric properties, such as refractive index ${ }^{3,7-10}$, 
glass transition temperature ${ }^{8,11-17}$, intrinsic viscosity ${ }^{18,19}$, solubility parameters ${ }^{20}$ and conformational property ${ }^{21}$. In this work we demonstrate the usefulness and focus of some of the parameters in deriving predictive QSPR models. The relation between the intrinsic viscosity[ $\eta]$ of poly(methyl methacrylate) and quantum chemical calculated parameters, LUMO, HOMO, energy gaps $(\Delta \mathrm{E})$, electronic energy, dielectric energy, $\omega$ and $\mu$ investigated theoretically.

\section{Modeling and Geometry optimization}

Full geometric optimization calculations for eight solvents (toluene, ethyl acetate, benzene, chloroform, butanone, dichloroethane, acetone and nitroethane) were performed using PC gamess ${ }^{22}$. Geometry optimizations were performed using 6-31G(d) basis set and PBE method ${ }^{23}$. Physical properties calculated involve electronic energy, dielectric energy, energy gaps $(\Delta \mathrm{E})$, LUMO energy and HOMO energy, $\omega$ and $\mu$. The experimental values of intrinsic viscosity[ $\eta]$ of poly(methylmethacrylate) of eight solvents taken from reference ${ }^{18}$. The structure of poly(methylmethacrylate) under study shown in Scheme 1.

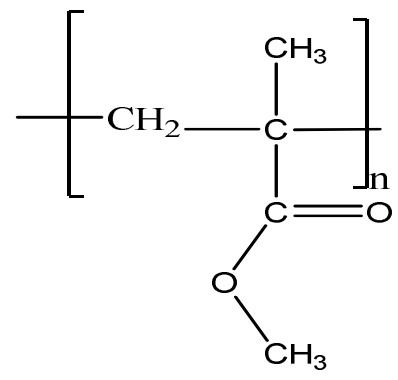

Scheme 1. Structure of poly(methylmethacrylate)

\section{Results and Discussion}

Multiple linear regressions (MLR) are one of the mathematical methods which have an extent application. The predictive model of QSPR study has been built up with the help of the following descriptors (Table 1). These descriptors for poly(methylmethacrylate) under study were calculated. The best model derived from the (MLR) analysis was used to intrinsic viscosity polymer in the eight organic solvents which is using in this study (toluene, ethyl acetate, benzene, chloroform, butanone, dichloromethane, acetone and nitro ethane). Several equations were generated by using all the variables and the best statistically model that we have obtained is four descriptor equations. To establish the statistical correlation, the physicochemical parameters were taken as independent variables and intrinsic viscosity as dependent variable. The best model was selected on the basis of statistical parameters viz observed with high correlation coefficient (R), sequential Fischer test $(\mathrm{F})$ and low standard error of estimate (SE), were employed to judge the validity of regression equation and evaluate the obtained QSPR models ${ }^{24-25}$. The model of QSPR study has been build up with help of the theoretical descriptors and experimental descriptors (Table 1). The best model derived from the (MLR) analysis was used to intrinsic viscosity polymer in the 8 organic solvents which is using in this study (toluene, ethyl acetate, benzene, chloroform, butanone, dichloroethane, acetone and nitroethane). Several equations were generated by using all the variables and the best statistical model that we have obtained is four descriptor equations ${ }^{26-30}$. 
Table 1. Calculated physicochemical descriptors of the solvents

\begin{tabular}{|c|c|c|c|c|c|c|c|c|c|}
\hline $\begin{array}{l}\text { No. } \\
\text { molecular }\end{array}$ & Solvents & $* \operatorname{Exp}[\eta]$ & $\begin{array}{l}\text { Elec. } \\
\text { Energy }\end{array}$ & $\begin{array}{l}\text { Dielec. } \\
\text { Energy }\end{array}$ & LUMO & HOMO & $\begin{array}{c}\text { E.Gap } \\
(\Delta \mathrm{E})\end{array}$ & $\mu$ & $\omega$ \\
\hline 1 & Toluene & 101 & -33906.2 & -0.416 & 0.866 & -10.435 & 11.301 & 4.784 & 2.025 \\
\hline 2 & Ethyl ac & 60 & -22184.7 & -0.776 & 0.77 & & & 4.9 & 2.128 \\
\hline 3 & Benz & 70 & -34535 & -0.397 & & & & & 2.025 \\
\hline 4 & Chls & 124 & & -0.6 & & & & & 2.093 \\
\hline 5 & & 49 & -16308.4 & -1.008 & & & & 4.9 & 2.173 \\
\hline 6 & Dichlo & 60 & -18517.1 & -0.921 & & & 11.3 & 4.9 & 2.144 \\
\hline 7 & & 48 & -15979.3 & -1.024 & 0.7 & & 11.406 & $4.9^{\prime}$ & 2.167 \\
\hline 8 & Nitroethane & 58 & -14631.8 & -1.054 & 0.726 & -10.691 & 11.417 & 4.982 & 2.174 \\
\hline
\end{tabular}

$* R e f=18, \operatorname{Exp}[\eta]=$ Experimental viscosity of polymer, $\triangle E=$ energy gaps in $\mathrm{eV}, \mathrm{HOMO}=$ The energy of highest occupied molecular orbital in $\mathrm{eV}, L U M O=$ The energy of lowest unoccupied molecular orbital in $\mathrm{eV}$, Elec. energy=electronic energy in $\mathrm{eV}$, Dielec. energy $=$ dielectric energy in $\mathrm{eV}, \mu=$ Chemical potential, $\omega=$ Electrophilicity index.

From Table 1, the model of QSPR study has been built up with help of the theoretical descriptors electronic energy, dielectric energy, energy gaps $(\Delta \mathrm{E})$, LUMO energy and HOMO energy, $\omega$ and $\mu$. The several QSPR model depends on the four theoretical descriptors Table 2.

Table 2. Statistical parameters of the linear regressions models obtained by using four descriptors

\begin{tabular}{cccc}
\hline Descriptors & $\mathrm{R}^{2}$ & $\mathrm{~F}$ & $\mathrm{~S}$ \\
\hline Elec E, $\omega$, Dielec.E \& E.Gap & 0.993 & 107.222 & 3.447 \\
Elec.E, LUMO, Dielec.E \& E.Gap & 0.992 & 102.395 & 3.527 \\
Elec.E, LUMO, Dielec.E \& HOMO & 0.992 & 102.395 & 3.527 \\
Elec.E, $\mu$, Dielec.E \& E.Gap & 0.992 & 98.259 & 3.600 \\
\hline
\end{tabular}

The best model was chosen, from Table 2 as the excellent model which it has less standard error (SE) and high $\mathrm{R}^{2}$ and $\mathrm{F}$ values. The model when depend on four theoretical descriptors Elec.E, $\omega$, Dielec.E and E.Gap, gave good model with correlation coefficient $\mathrm{R}^{2}$ values for this model of 0.993 , as equation 1 .

$[\eta]=0.1308$ Elec.E-456.918 $\omega+2651.095$ Dielec.E-6922.180E.Gap+84796.039

In Eq. 1 the positive signs of Elec. E and Dielec. E descriptors refers to a positive correlation with the intrinsic viscosity while negative signs of $\omega$ and E.Gap descriptors suggest that the intrinsic viscosity decreases with increasing value of this descriptor. The relationship between the experimental and predicted data in the model represented in the Figure 1.

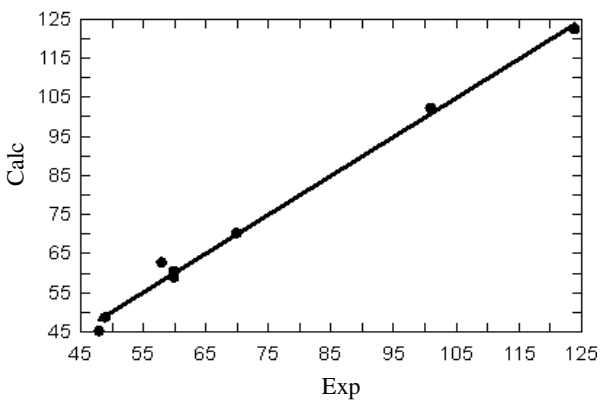

Figure 1. Plot of intrinsic viscosity prediction vs. experimental intrinsic viscosity using Eq.1 
In Eq 2, the negative sign of E.Gap descriptor refers to inverse correlation between E.Gap with the intrinsic viscosity and on the other hand in this model E.Gap have negative sign which suggests that the intrinsic viscosity increases with decreasing value of this parameter. The relationship between the experimental and predicted data shown in Figure 2 .

$[\eta]=0.1316$ Elec.E+371.825LUMO+2669.675Dielec.E-7088.728E.Gap+85465.143

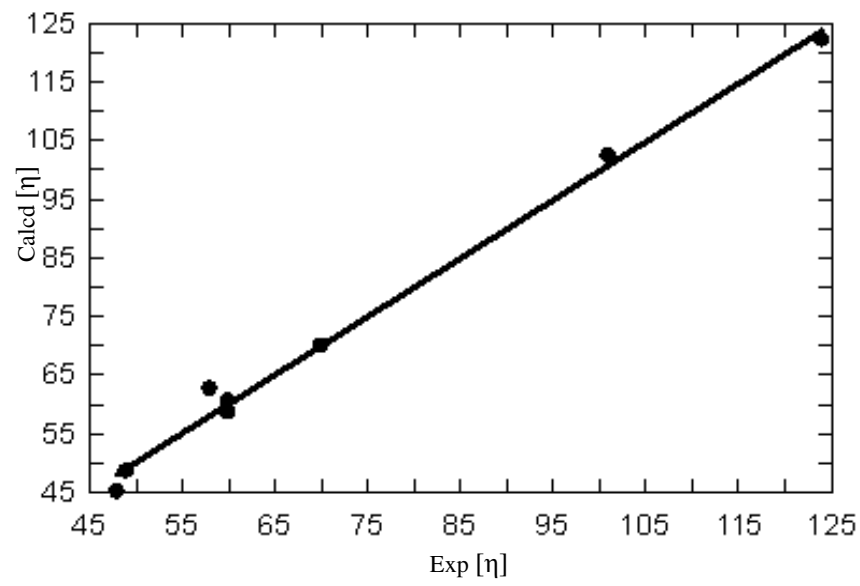

Figure 2. Plot of intrinsic viscosity prediction $v s$. experimental intrinsic viscosity using Eq.2

In Eq. 3 the positive signs of Elec.E, Dielec.E and HOMO descriptors refers to a positive correlation with the intrinsic viscosity while negative signs of LUMO descriptors suggest that the intrinsic viscosity decreases with increasing value of this descriptor. The relationship between the experimental and predicted data is represented in the Figure 3.

$[\eta]=0.1316$ Elec.E-6716.903 LUMO+2669.675 Dielec.E+7088.728 HOMO+85465.143

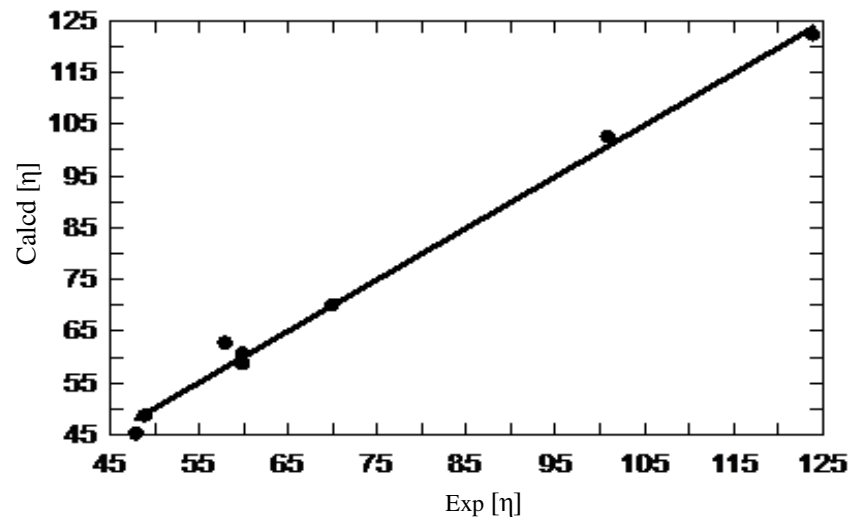

Figure 3. Plot of intrinsic viscosity prediction $v s$. experimental intrinsic viscosity using Eq.3

While Eq. 4 the positive signs of Elec.E, Dielec.E descriptors refers to a positive correlation with theintrinsic viscosity while negative signs of $\mu$ and E.Gap descriptors suggest that the intrinsic viscosity decreases with increasing value of this descriptor. The relationship between the experimental and predicted data is represented in the Figure 4.

$[\eta]=0.132$ Elec.E-358.156 $\mu+2681.347$ Dielec.E- 6934.762E.Gap+85780.327 


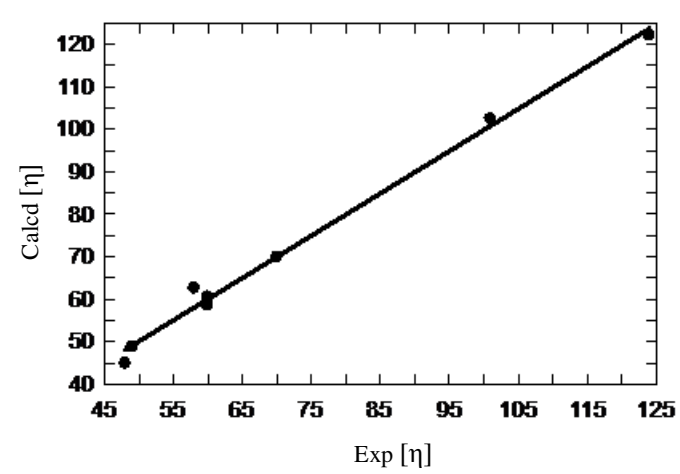

Figure 4. Plot of intrinsic viscosity prediction vs. experimental intrinsic viscosity using Eq.4

\section{Conclusion}

The present study have been shown to provide very good QSPR models for the estimation of intrinsic viscosity of poly(methyl methacrylate) solution by using values theoretical descriptors calculated. The study indicated that intrinsic viscosity[ $\eta]$ of poly(methyl methacrylate) solution parameters of organic solvents can be modeled. The best model when depends on theoretical descriptors [Elec.E, $\omega$, Dielec.E and E.Gap] was Eq.1, From all the results, the QSPR models, Eq. 1. And this showed insignificant role in predict the QSPR model of the intrinsic viscosity[ $\eta]$ of poly(methylmethacrylate) solution. This result encourages the application of QSPR techniques to awider selection of polymer properties.

\section{References}

1. Camarda K V and Maranas C D, Ind Eng Chem Res, 1999, 38(5), 1884-1892; DOI:10.1021/ie980682n

2. Katritzky A R, Sild S, Lobanov V and Karelson M L, J Chem Inf Comput Sci, 1998, 38(2), 300-304; DOI:10.1021/ci9700687

3. Katritzky A R, Sild S and Karelson M, J Chem Inf Comput Sci, 1998, 38(6), 11711176; DOI:10.1021/ci980087w

4. Bicerano J, Prediction of polymer properties. $2^{\text {nd }}$ Ed., New York: Marcel Dekker; 1996,

5. Yao X J, Wang Y W, Zhang X Y, Zhang R S, Liu M C, Hu Z D and Fan B T, Chemom Intell Lab Syst., 2202, 62(2), 217-225; DOI:10.1016/S0169-7439(02)00017-5

6. $\mathrm{Xu}$ J, Guo B, Chen B and Zhang Q, J Mol Model. , 2005, 12(1), 65-75; DOI:10.1007/s00894-005-0006-X

7. Xu J, Zheng Z, Chen B and Zhang Q, QSAR Comb Sci., 2006, 25(4), 372-379; DOI:10.1002/qsar.200530143

8. García-Domenech R and de Julián-Ortiz J V, J Phys Chem B, 2002, 106(6), 15011507; DOI:10.1021/jp012360u

9. $\mathrm{Xu}$ J, Chen B, Zhang $\mathrm{Q}$ and Guo B, Polymer, 2004, 45(26), 8651-8659; DOI:10.1016/j.polymer.2004.10.057

10. Yu X, Yi B and Wang X, J Comput Chem., 2007, 28(14), 2336-2341; DOI:10.1002/jcc. 20752

11. Cao C and Lin Y, J Chem Inf Comput Sci., 2003, 43, 643-650.

12. Afantitis A, Melagraki G, Makridima K, Alexandridis A, Sarimveis H and IglessiMarkopoulou O, J Mol Struct., Theochem., 2005, 716(1-3), 193-198; DOI:10.1016/j.theochem.2004.11.021 
13. Simona Funar-Timofei, Smaranda Iliescu, MATCH Commun Math Comput Chem., 2008, 60, 997-1005

14. Kawkab Ali Hussain, Iraqi National J Chem., 2013, 49, 47-60.

15. Bertinetto C, Duce C, Micheli A, Solaro R, Starita A and Tiné M R, Polymer, 2007, 48(24), 7121-7129; DOI:10.1016/j.polymer.2007.09.043

16. Duce C, Micheli A, Starita A, Tiné M R and Solaro R, Macromol Rapid Commun., 2006, 27(9), 711-715; DOI:10.1002/marc.200600026

17. Duce C, Micheli A, Solaro R, Starita A and Tiné M R, Macromol Symp., 2006, 234(1), 13-19; DOI:10.1002/masy.200650203

18. Afantitis A, Melagraki G, Sarimveis H, Koutentis P A, Markopoulos J, IgglessiMarkopoulou O, Polymer, 2006, 47, 3240-3248; DOI:10.1016/j.polymer.2006.02.060

19. Gharagheizi F, Comput Mater Sci., 2007, 40(1), 159-167; DOI:10.1016/j.commatsci.2006.11.010

20. Yu X, Wang X, Wang H, Li X and Gao J, QSAR Comb Sci., 2006, 25(2), 156-161; DOI:10.1002/qsar.200530138

21. Yu X, Yi B, Xie Z, Wang X and Liu F, Chemometr Intell Lab Syst., 2007, 87(2), $247-$ 251; DOI:10.1016/j.chemolab.2007.03.001

22. Schmidt M W, Baldridge K K, Boatz J A, Elbert S T, Gordon M S, Jensen J H, Koseki S, Matsunaga N, Nguyen K A, Su S J, Windus T L, Dupuis M and Montgomery J A, J Comput Chem., 1993, 14(11), 1437-1463; DOI:10.1002/jcc.540141112

23. a) Perdew J P, Burke K and Ernzerhof M, Phys Rev Lett., 1996, 77(18), 3865-3868; DOI:10.1103/PhysRevLett.77.3865 b) Ernzerhof M and Scuseria G E, J Chem Phys., 1999, 110, 5029-5036; DOI:10.1063/1.478401

24. S. M-H Ismael, K.A. Hussain and Matter A D, Chem Sci Trans., 2015, 4(2), 355-360; DOI:10.7598/cst2015.1002

25. Kawkab Ali Hussain, Sadiq M H Ismael and Wisam A Radhi, British Journal of Pharmaceutical Research, 2016, 13(1), 1-11

26. Shailja Sachan, Santosh Tiwari and Santosh Kumar Tiwari, Int J Pharm Tech Res., 2014, 6(5), 1687-1694.

27. Mehta Parul D and PathakA K, Int J Pharm Tech Res., 2012, 4(2), 676-688.

28. Supratim Ray, Int J ChemTech Res., 2012, 4(1), 41-47.

29. Fakhr M Abu-Awwad, Int J ChemTech Res., 2009, 1(3), 742-750.

30. Margabandu R and Subramani K, Int J ChemTech Res., 2010, 2(3), 507-1513. 